\title{
The use of performance indicators for environmental assessment within the railway business: the RAVEL workbench prototype, a web-based tool
}

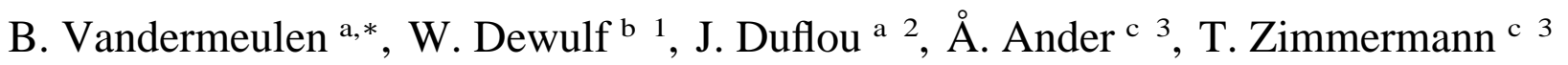 \\ ${ }^{a}$ Centre for Industrial Management, Katholieke Universiteit Leuven, Celestijnenlaan 300A, B3001 Heverlee, Belgium \\ ${ }^{\mathrm{b}}$ Katholieke Universiteit Leuven, Afdeling Productietechnieken, machinebouw en automatisering (PMA), Celestijnenlaan 300B, \\ B3001 Heverlee, Belgium \\ c Bombardier Transportation, Department of ICT/K, SE-721 73, Västerås, Sweden
}

Received 26 November 2001; accepted 14 October 2002

\begin{abstract}
Within the Brite/Euram project RAVEL, an Internet-based tool has been developed which allows an assessment of railway vehicles by means of a set of environmental performance indicators (EPIs). The specific methodology that is applied for such an assessment is highlighted in this article, along with consideration of the feasibility of system implementation within an industrial environment. The way this methodology is applied is illustrated by, on the one hand, a description of the prototype for the webbased RAVEL tool, and on the other hand, by the calculation of EPI scores for an exemplary train system. Finally, the possibility of extending the practise to other industrial sectors is explored.

(c) 2002 Elsevier Science Ltd. All rights reserved.
\end{abstract}

Keywords: Environmental performance indicator; (Web-based) design for environment tool; Railway industry

\section{Introduction}

RAil VEhicLe eco-efficient design (RAVEL) is a European project that has been focussing on the development of a design for environment (DfE) system for railway vehicles [1]. Involved in the project were a train manufacturer (Bombardier Transportation), the Danish and Swedish railway operators (DSB and SJ), a supplier of rubber subsystems (Woodville Polymers), consultancy companies (ABB and GEP) and universities (Chalmers University, Linköping University and the Katholieke Universiteit Leuven). The project was

* Corresponding author. Tel.: +32-16-322760; fax: +32-16-322986.

E-mail addresses: bert.vandermeulen@cib.kuleuven.ac.be (B. Vandermeulen); wim.dewulf@mech.kuleuven.ac.be (W. Dewulf); joost.duflou@cib.kuleuven.ac.be (J. Duflou); asa.ander@se.transport.bombardier.com (. Ander); tobias.zimmermann@ch.transport.bombardier.com (T. Zimmermann).

1 Tel.: +32-16-322512; fax: +32-16-322987

2 Tel.: +32-16-322845; fax: +32-16-322986

3 Tel.: +46-21-322639; fax: +46-21-128803 initiated because of the need for appropriate support tools when trying to enhance the eco-efficiency of railway vehicles. Also the need for (international) standardisation of environmentally related evaluation criteria, that can be included in tenders and sales contracts, was recognised. Hence, the overall RAVEL objective was to develop a workbench consisting of a set of tools that enable designers to improve the eco-efficiency for railway vehicles during their entire life cycle.

The RAVEL project is supposed to be a first major step towards the introduction of an environmental performance indicator (EPI) standard in the railway sector. The proposed indicators (listed in Table 1) are currently under consideration for adaptation by the UIC. ${ }^{4}$

Improved eco-efficiency is an increasingly important marketing feature for vehicle manufacturers in the railway sector and for competition with other transportation sectors such as car industry and regional airlines. The

\footnotetext{
${ }^{4}$ Union Internationale des Chemins de fer-International Union of Railways-Internationaler Eisenbahnverband.
} 
Table 1

The 15 implemented EPIs, with their abbreviations and units

\begin{tabular}{|c|c|c|}
\hline No. & EPIs & Abbreviations \\
\hline 1 & Amount of prohibited materials $(\mathrm{kg})$ & APM \\
\hline 2 & Amount of restricted materials $(\mathrm{kg})$ & ARM \\
\hline 3 & Component complexity (ratio) & $\mathrm{CCX}$ \\
\hline 4 & $\begin{array}{l}\text { Cradle-to-gate material index (Eco Ind. } \\
99)^{\mathrm{a}}\end{array}$ & CMI \\
\hline 5 & Degree of inventoried materials (wt $\%$ ) & DIM \\
\hline 6 & $\begin{array}{l}\text { Existence of disposal/recycling manuals } \\
\text { (wt\%) }\end{array}$ & DRM \\
\hline 7 & Fraction recycled materials (wt $\%$ ) & FCM \\
\hline 8 & Fraction renewable materials (wt\%) & FRM \\
\hline 9 & Fraction reused components (wt $\%$ ) & FRC \\
\hline 10 & $\begin{array}{l}\text { Marking of selected material and } \\
\text { component groups }(\mathrm{wt} \%)\end{array}$ & MSG \\
\hline 11 & Possible hazardous waste rate (wt\%) & PHW \\
\hline 12 & Possible material recycling rate (wt\%) & PMR \\
\hline 13 & $\begin{array}{l}\text { Suppliers with EMS (environmental } \\
\text { management system) (wt\%) }\end{array}$ & EMS \\
\hline 14 & System mass (kg/seat-km) & SYM \\
\hline 15 & Total energy consumption ( $\mathrm{kW} \mathrm{h/seat-km)}$ & TEC \\
\hline
\end{tabular}

${ }^{\text {a }}$ Eco-indicator 99 points.

importance of offering environmental friendly means of transportation is further stressed by the increasing overall transport volumes and society's environmental awareness. The indicators that have been developed as part of the RAVEL methodology try to offer an objective comparison between competing solutions.

\section{RAVEL concepts}

\subsection{Methodology}

The assessment method developed in the RAVEL project differs from traditional LCA methods, because it is completely based on performance indicators as a measure for the eco-efficiency of the product. The main advantage of this approach is that environmental coordinators and project managers can calculate and evaluate indicator scores for very specific environmental aspects. In this way, they can actually customise the assessment according to their own priorities and policies, or tune it to the customer's requirements. However, this flexibility adds complexity to the system which makes it more vulnerable to misuse and abuse, e.g. by omitting indicators for certain parts of the product where a rather negative score is expected. Basically, the system is built on the same principles as an environmental management system like ISO 14001 or EMAS (Eco Management and Audit Scheme). This means, it identifies significant environmental design aspects, includes target setting and allows performance measurement. In general, it provides a catalyst for continuous improvement applicable to pro- duct development processes as encountered in today's railway business.

Fig. 1 gives a more process-based view on the RAVEL methodology. It shows the operator and a manufacturer communicating by means of tenders during the process of ordering and designing a rail vehicle. The RAVEL support helps them performing the tasks. Also the internal RAVEL processes, like creating designs and calculating EPIs, are displayed. Note that in general, these processes are not straightforward. Several iterations might be necessary to obtain an acceptable solution. The train manufacturer, for example, may conclude that after creating designs and calculating EPI scores, the result is still below target, so there has to be a redesign phase, followed again by the calculation of EPI scores. This scenario can be repeated an unlimited number of times until all targets have been reached.

The methodology is designed such that it is possible to select a subcomponent of the product and calculate any of up to 15 available indicators: the EPIs that are implemented in the current RAVEL prototype. A powerful feature of the system is that designers - as users of the workbench - can compare the scores of alternative component designs, and thus select the most appropriate solution to fulfil the function.

\subsection{Data structure}

The products considered are railway vehicles or vehicle parts. These vehicles are entities with a long anticipated life span. They are composed of a large number of (sub)components, containing several hundreds of different materials. At first glance, it could be assumed that such complexity would surely cause data problems. To calculate the EPI scores, underlying data is required. Two kinds of data are being distinguished. On one hand, there are data linked to a specific project or component, which we call product data. On the other hand, the database also contains basic (environmental) data, referring to known physical, chemical and environmental properties of a wide range of materials.

Data gathering and data input are the main resource consuming tasks when using the workbench. To facilitate the process of data input, it is planned to link the RAVEL database with company's existing product data management (PDM) systems.

\subsection{Indicators}

The use of performance indicators to measure business performance has been strongly adopted by modern management $[2,3]$. The focus has, however, mainly been on the economic efficiency of operational (factory floor related) and logistic processes. In recent years, the interest in environmental performance of products during their entire life cycles has been growing. Today, there 


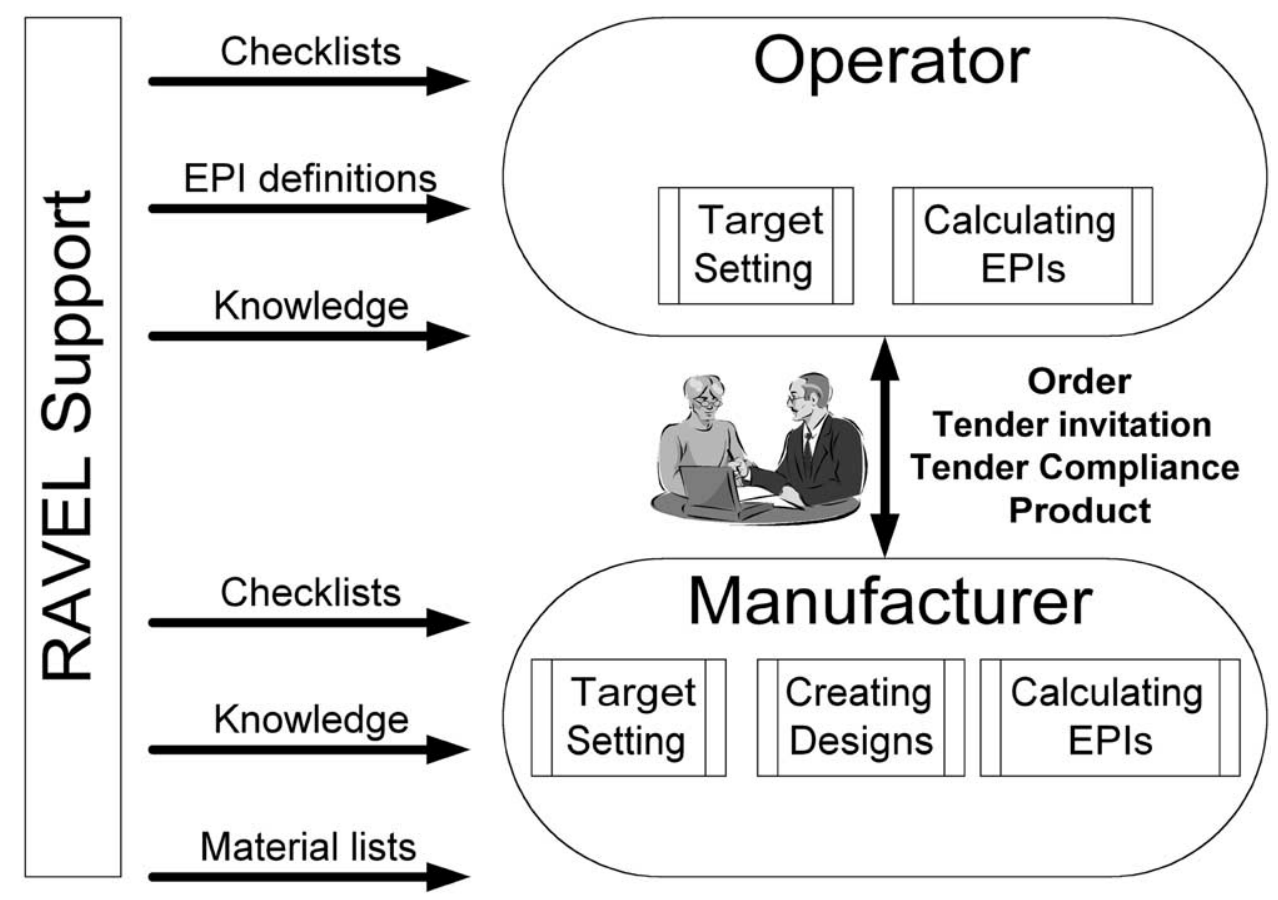

Fig. 1. Representation of the RAVEL methodology. It shows communication between the operator and the manufacturer, the internal processes and support.

is a pressing need for standardised EPIs that support comparative environmental performance evaluations [4].

To avoid ambiguity and linguistic vagueness, quantified EPIs were preferred by the RAVEL project team. Most EPIs are therefore, figures resulting from calculations for which the environmental data provided in the database serves as input. Each indicator is well defined and its value is bound to a known but indicator dependent interval. These characteristics provide the possibility of transforming the scores towards a uniform interval (normalisation) and to apply weighting to obtain an overall score. However, merely due to the fact that it implies subjective assignment of weighting factors, no default weighting system was included in the workbench.

As stated in the overall objective of the RAVEL project, the set of indicators is developed so that attention is drawn to each of the three life cycle phases: production, use and disposal. The indicators can be divided into categories like: 'materials', 'manufacturing and assembly', 'energy', 'emissions from wear', 'air pollution during use', 'maintenance' and 'end-of-life'. Also other environmental aspects like noise and emissions were recognised as important impact factors, but were not included.

An example of an indicator from the category materials is the 'amount of restricted materials'. This indicator quantifies the total amount of materials that are on a (predefined) black list and found within the material inventory of the product tree.
Before indicators are calculated, target scores are preset. By setting quantitative target values for each selected indicator, the desired environmental performance of a vehicle is already fixed before the actual design work starts. This is the control value towards which the designers should aim. For example, these targets can originate from EPI scores from a well-defined previous project (called 'the baseline'), increased with a certain percentage of desired improvement, or from requirements imposed by customers or legislation. While some indicators can be applied to implement a strategy of incremental improvements, others can serve as hard reject criteria. For example, in the case of the 'amount of prohibited materials' (APM) indicator, a rigid zero target value could be imposed.

\subsection{Communication}

To support the communication between customers and suppliers, operators and manufacturers, the tender process is incorporated in the workbench.

Contrary to most off-the-shelf products, the ordering of trains is a process with intensive cooperation between operators, manufacturers and suppliers of subsystems and materials. On one hand, this opens the possibility for the client (operators) to state specific requirements and expected deliverables in line with their corporate viewpoints. The tender workflows inside the RAVEL system allow specifying the environmental needs by means of the EPI target values plus additional require- 
ments. On the other hand, these specifications can serve as a reference anchor and comparability check for the manufacturers themselves. Under these circumstances, a common environmental language has become indispensable.

After competing manufacturers have submitted their compliance forms, the operator can compare and evaluate them.

\section{Functional description of the RAVEL workbench prototype}

\subsection{Introduction}

The RAVEL workbench prototype is yet a complex but powerful web-based tool. The main effort has been invested in showing functionality to future end users, providing a good perspective on what will be possible when a more professional and robust version becomes available in a later stage. The database can be reached through the Internet so the users, no matter where they are located, can always communicate with the system. Modifications are carried out in real-time and multiple users can work simultaneously, directly interacting on the changes made by others.

The workbench consists of three big workspaces: the project workspace, the component workspace and the knowledge system (KS). The tender process-as mentioned earlier-is included as a separate part of the workbench to facilitate communication between business partners.

\subsection{Project workspace}

This part of the system is the workspace for the project manager or the environmental coordinator. Here, the project structure can be designed and displayed in a tree view. The first part is a Checklist that helps the user to find his way through the workflow. The Admin (project administration) part allows setting up the system tree of the train (or a specific part of it). New projects can be started here and existing projects modified. The next step is to enter the project's Basic Data. This is in fact general information about the project, such as the name of the responsible persons, the impact indication principle (IIP), and so on. This IIP unambiguously refers to the way the EPIs are defined. ${ }^{5}$ Indicator scores are only comparable if the same IIP is used. Parameters are pieces of information about the product, e.g. the number of seats in a vehicle or the expected product lifetime. These are

${ }^{5}$ Within the same IIP, all (mathematical) definitions of the indicators are fixed. An IIP is characterised by a date and version number and should be defined by a central institution. This anticipates future upgrading of the EPI formulas. important because they are used in calculations that normalise the EPI results. The Indicators part is used to specify which indicators will be used throughout the project. The project managers can select any of the 15 available EPIs to make the assessment. With the indicators selected, the user can now enter Target values for them. For most projects, it is assumed that reference can be made to some baseline values or requirements by customers or legislation. The user can also consult the KS for help with the target setting. Once each subsystem has a target, these can be aggregated automatically to the higher (parent) system. On the other hand, a target of a large system can be broken down towards the subsystems-which are a more common problem designers will have to deal with.

The Design part actually links a physical component from the database with a logical (sub)system. Several similar components can be selected as designs in this way. The user can compare the different options and indicate which one should preferably be withheld for further calculations. With the IIP specified, the indicators selected, and the designs linked, all prerequisites for performing Calculations have been met. The result of the EPI calculation is a tabulated overview of the results. The output generated here is much more than only the EPI score; also, for convenience, the intermediate results, assumptions and parameters are given. The Internet browser's print function can be used to create a hardcopy.

\subsection{Component workspace}

The component workspace is the place where engineers can declare and modify the train components. These components are projects independent because the same component can appear in different projects. Property data of all kinds (physical, chemical, environmental, economical, etc.) can be entered. The inventory tool is the most important tool here. It offers engineers the possibility to work on the structure of the different train components. They can alter or substitute the used materials and their quantities. Different components can be linked together to constitute bigger units: compositions. The act of making a composition can also bring along properties that did not belong to any of the lower structures.

\subsection{Knowledge system}

The KS is an important part of the workbench because it provides the user with the necessary support to perform specific tasks. It fulfils a (software) help and knowledge support function at the same time. From our conversations with designers involved in the RAVEL project, we learned that they do not have the tendency to look up knowledge inside computer systems. A sceptical 
attitude towards such novelties can be observed. They want things to be done fast and therefore depend on their experience and intuition most of the time, occasionally consulting colleagues on the matter. As a consequence, the introduction of KSs requires an explicit effort and cooperation of all involved people. The knowledge base already available in the RAVEL workbench prototype can provide a useful stepping stone in this respect.

Throughout the whole RAVEL workbench system, the graphical user interface contains several 'KS-buttons', leading directly to relevant information. This approach could be called situation-based support; by evaluating the circumstances under which a user calls for help, knowledge support is guaranteed to be very concise and to-the-point.

The KS helps designers and managers with setting environmental targets, finding alternative design solutions, sharing their experiences and so on.

\subsection{User types}

Although not completely implemented in the prototype version, the RAVEL system was designed to differentiate between types of users. The login information will ensure that the interface only shows those parts, which correspond to the user's access rights, as set by the system administrator, while hiding the other ones. Different possible user types are: operator, project manager, designer, environmental coordinator and engineer. Yet another distinction is made from a supply chain point of view. A train manufacturer can act as a customer while buying raw materials or subsystems, but as a supplier while selling the train. The operators will always be considered as customers buying trains. These distinctions have far-reaching consequences concerning the use of the RAVEL workflow.

\section{A RAVEL example}

In this part, a practical example of how the RAVEL methodology may work in practise is described. Although the sketched situation, train project and EPI results are fictional, all methods and figures were chosen to be as realistic as possible. The main purpose of this example is to illustrate the procedures chronologically, from tender process until the final order. Therefore, it might sound sometimes as if the RAVEL system is already in use, which is not the case. In fact, robust software implementation will be covered by a follow-up project called REPID, requested by the UCI and UNIFE associations.

When a train operator wants to buy a series of new vehicles, a tender invitation is sent out to different manufacturers, stating the requirements. Once the answers to the tender invitation have been received, they need to be compared and ranked. This ranking can be done manually, based on the operator's own perception. At all times, the chosen manufacturer will have to verify and secure his compliance to the promised environmental performance. That is the reason why both manufacturer and operator base their performance evaluation on the same (standardised) RAVEL indicator system.

In practice, the tender process-where the customer evaluates the manufacturer-lies in between a preliminary product platform development and the actual order processing, and generally takes about two months. During the platform development as well as the order processing, three types of design activities can be distinguished: the conceptual, the preliminary and the detailed design. RAVEL was intended to support all three phases. During the conceptual design phase, data must be gathered from old databases, e.g. previous design project databases. In the detailed design phasewhich this example is about-subsuppliers are contacted and in-house knowledge may be more up to date, offering a better perspective.

At the manufacturers' side, first all required datacomponent and material inventory data-are collected and inserted. The project structure is then set up. The first tree level contains the systems: car body, bogies and bogie mounted brakes, power supply, propulsion, auxiliaries, brakes, interiors and others.

The second tree level is entered and each subproject is linked to the corresponding physical component present in the database. Basic data and parameters, such as the components' masses and expected lifetimes, are filled in. This method allows variations of a component to be constructed without having to enter all of the information every time.

Next, indicators have to be selected. Suppose the management of the train building company wants to do a simple assessment, only calculating the indicators most important for them. The EPI 'total energy consumption' (TEC) could be chosen as a first indicator from the most recent IIP set. Energy use is known as a universal measure for efficiency and has a clear relation with environmental consequences as soon as the origin of the energy is known. Wind, hydro- and solar-energy are supposed to be less harmful than energy generated through the incineration of fossil fuels. So, depending on the energy source(s), the importance given to this EPI can vary. ${ }^{6}$ The second EPI to be looked at could be 'fraction renewable materials' (FRM), e.g. because of the company's environmental policy which dictates that extra attention has to be paid to renewables. (This could be the case,

\footnotetext{
${ }^{6}$ The approach to energy use as outlined here is a simplified one. In reality different countries might sell excess energy to each other and a more complex model including marginal effects (what happens if we use one energy unit more or less?) may be needed in order to obtain a trustworthy assessment.
} 
in order to comply with the past advertising campaign, which was focussed on creating an edge over competitors concerning green image.) Renewable materials are for example plywood, wool and leather.

The third EPI, APM is chosen due to the fact that both national and European legislation is becoming tighter with regards to this subject. No materials (on the government's forbidden list) are allowed; the result has to be $0 \mathrm{~kg}$.

Although most indicators are expressed in units that make them independent of scale and use factors-e.g. through dividing by the number of seats and kilometres, or by expressing them as weight percentage-choosing both challenging and achievable target value remains a difficult task. To help the user with this, instant links to the KS is supposed to assure high quality support at all times. In the case of APM the target setting is easy, it is $0 \mathrm{~kg}$. For the other two indicators, it is more difficult. Management agrees to improve the EPI compared to the baseline value of $0.04 \mathrm{~kW} \mathrm{~h} /$ seat-km with $5 \%$ to 0.038 $\mathrm{kW} \mathrm{h} /$ seat-km for the total energy indicator. The fraction of renewable materials has to improve even more: from 2.5 to $3.5 \mathrm{wt} \%$ (weight percentage).

In order to reach these targets, designers will have to implement some significant improvements. On a project management level, the overall train targets can be split up for the various subparts, taking into account which parts have the greatest potential for improvement. In our example, it was found that the best strategy for reaching the desired amount of renewable materials lies in redesigning the train's interior. After being confronted with this problem, the interior designer claimed that it would be hard to do, because classical interior designs barely reached a FRM of $3 \mathrm{wt} \%$. Consulting the KS learned that, for example, replacing the plastic seat covering with natural fibre clothing is a possibility. Designers can always propose alternative designs with better EPI scores, but other aspects, like e.g. fire safety, must also be analysed. In the end, if the cost of the new design is higher, it will be up to the management to decide whether the environmental improvements are worth it or not. An example of this polarity between traditional requirements and environmental requirements is the well known problem that, on the one hand, bromine is often found on the list ${ }^{7}$ of restricted materials, while on the other hand, the best flame retardants are often brominated substances. The scores of the indicators were calculated. The amount of prohibited materials inside the train was indeed found to be $0 \mathrm{~kg}$, which equals the target value.

The FRM was calculated and a value of $3.6 \mathrm{wt} \%$ was

\footnotetext{
${ }^{7}$ Different lists may exist and be used within different companies, industries or countries. At the moment, standardised lists are not present.
}

obtained, doing slightly better than the minimal requirement. When looking at the different train parts, the interior had the highest FRM score $(9.9 \mathrm{wt} \%)$. This score was reached through the use of wool, cardboard, plywood and leather in, amongst others, passenger seats and interior lining.

The value for the TEC was found to be $0.032 \mathrm{~kW}$ $\mathrm{h} / \mathrm{seat}-\mathrm{km}$, merely due to the decreased total vehicle mass and some less energy consuming auxiliaries.

Once the train data are present inside the database, it does not require too much time to perform the EPI calculations. Each result was found within less than 1 $\min ;{ }^{8}$ of course this depends mainly on the server speed and the network connection used.

When all calculations are finished and the results have been verified to be in compliance with the targets, the RAVEL DfE cycle is complete. However, it can be very useful at this point to document the encountered difficulties and their solutions, to serve as input for projects to come. This is typically the task of an environmental coordinator. The KS provides a range of functions to enter this new information into the knowledge base. The EPI scores can be communicated to the customer (who will verify the data) and production can start after acceptance of the design proposal.

\section{Future}

\subsection{Acceptance of the RAVEL results}

Comments and feedback received during testing and validation of the RAVEL prototype workbench indicate that the system may get broad acceptance and be widely used, once a robust, commercial software version becomes available. As with all new methods of evaluation, the RAVEL system will take time to be adopted and used to its maximum potential.

At the beginning of the RAVEL project, it was already expected that the methodology could only be applied successfully, if it was computer-based, fast, intuitive and (at least partly) automated. Therefore, one important requirement for the methodology was that, whenever achievable, processes, procedures and definition of information should be formalised to allow for computer implementation. A more accessible user interface, along with a better integration into the company's existing (data) structures is needed in order to further increase the ease-of-use.

Apart from software requirements, also standardisation is an indispensable success criterion. If the RAVEL methodology is accepted as a standard, interest from rail-

\footnotetext{
${ }^{8}$ Using a Pentium I, $133 \mathrm{MHz}$ computer, Microsoft Internet Explorer 5.5 ${ }^{\mathrm{TM}}$
} 
way business related companies is expected to increase even more, eventually becoming a necessity not in order to be excluded from tendering procedures.

\subsection{Applicability outside the railway industry}

The authors believe that the methodology is extendable to other industries than the railway business. By making some small changes, the EPIs can be adapted to other products than trains. Most of them are based on basic material properties that are not specific to any sector, which gives them a universal characteristic. As long as the complete material inventory and component structure is known, these EPIs can be calculated. However, some EPIs may become irrelevant and should be discarded or replaced.

Besides trains, busses, aeroplanes and even buildings could also be analysed using the RAVEL methodology. It is true that in these industries, some environmental analysis tools have already emerged. The European Environmental Agency (EEA) [5] lists two categories of decision support systems (DSS): computer-based models in integrated environmental assessment (IEA) and computer intelligent processing technologies (CIPTs). The technical report by Peirce [6] gives an overview of IEA tools before 1998 .

A prerequisite to successful implementation of such a system is that the companies are convinced of the need for DfE and have a clear environmental policy. All designers, project managers and associated technical experts should have a general understanding about DfE, on the manufacturer's side as well as on the subsupplier's and operator's side. Also, new personnel may need to be educated and trained.

\section{Conclusions}

The RAVEL project has developed a methodology which allows the support of the various design phases of railway vehicles from an environmental perspective. EPIs are used to compare environmental impact and play an important role as information carriers in communication between stakeholders in the railway industry and towards the public. The RAVEL methodology has been implemented in web-based prototype software that also has been tested and validated. At the top level, three processes, which are supported by the prototypes can be distinguished:

1. translation of environmental requirements into quantitative targets;

2. calculation and verification of environmental performance;

3. supporting users with adequate knowledge and experience.

Test results are promising for the rail sector and the methodology can possibly be adapted to other similarly structured industries, helping companies in their quest for production of more sustainable goods.

\section{References}

[1] Dewulf W, Duflou J, Ander Å. Integrating eco-efficiency in rail vehicle design. Leuven, Sweden: Leuven University Press, 2001.

[2] Harvard Business Review. Harvard business review on measuring corporate performance. Harvard Business School Press, Boston, MA, USA; August 1998.

[3] AusIndustry. Key performance indicators manual: a practical guide for the best practice development, implementation and use of KPIs. London: Pitman, 1999.

[4] Carlson R, Forsberg P, Dewulf W, Karlson L, Ravemark D, Ander $\AA$. A full design for environment (DfE) data model. In: Proceedings of PDT Europe. Brussels, April 24-26. 2001. p. 129-35.

[5] The European Environmental Agency (EEA). 2001. Available from: http://service.eea.eu.int/envirowindows/dss.shtml.

[6] Peirce M. Computer-based models in integrated environmental assessment; a report produced for the European Environment Agency. Technical report no. 14; February 1998. 